\title{
Article \\ Health-Related Quality of Life following Cytoreductive Radical Prostatectomy in Patients with De-Novo Oligometastatic Prostate Cancer
}

\author{
Michael Chaloupka ${ }^{1}$, Lina Stoermer ${ }^{1}$, Maria Apfelbeck ${ }^{1}$, Alexander Buchner ${ }^{1}{ }^{\circledR}$, Vera Wenter ${ }^{2}$, Christian G. Stief ${ }^{1}$, \\ Thilo Westhofen ${ }^{1,+}$ and Alexander Kretschmer ${ }^{1, *,+}$ \\ 1 Department of Urology, LMU Klinikum, Ludwig-Maximilians University Munich, 81377 Munich, Germany; \\ michael.chaloupka@med.uni-muenchen.de (M.C.); L.Stoermer@campus.lmu.de (L.S.); \\ maria.apfelbeck@med.uni-muenchen.de (M.A.); alexander.buchner@med.uni-muenchen.de (A.B.); \\ christian.stief@med.uni-muenchen.de (C.G.S.); thilo.westhofen@med.uni-muenchen.de (T.W.) \\ 2 Department of Nuclear Medicine, LMU Klinikum, Ludwig-Maximilians University Munich, \\ 81377 Munich, Germany; vera.wenter@med.uni-muenchen.de \\ * Correspondence: alexander.kretschmer@med.uni-muenchen.de; Tel.: +49-89-44007-2971; \\ Fax: +49-89-44007-8890 \\ + Both authors contributed equally.
}

check for updates

Citation: Chaloupka, M.; Stoermer, L.; Apfelbeck, M.; Buchner, A.; Wenter, V.; Stief, C.G.; Westhofen, T.; Kretschmer, A. Health-Related Quality of Life following

Cytoreductive Radical Prostatectomy in Patients with De-Novo Oligometastatic Prostate Cancer. Cancers 2021, 13, 5636. https: / / doi.org/10.3390/cancers13225636

Academic Editor: Christian Thomas

Received: 28 October 2021

Accepted: 5 November 2021

Published: 11 November 2021

Publisher's Note: MDPI stays neutral with regard to jurisdictional claims in published maps and institutional affiliations.

Copyright: (c) 2021 by the authors. Licensee MDPI, Basel, Switzerland. This article is an open access article distributed under the terms and conditions of the Creative Commons Attribution (CC BY) license (https:// creativecommons.org/licenses/by/ $4.0 /)$.
Simple Summary: Recent retrospective data indicate a survival benefit of surgical removal of the prostate in patients with oligometastatic prostate cancer as part of a multimodal therapeutic regime. However, the impact of radical prostatectomy (RP) on patient's health-related quality of life (HRQOL) in this scenario has not been evaluated yet. In a contemporary and well-balanced cohort, we compared the self-assessed HRQOL of patients with oligometastatic prostate cancer with patients with locally confined prostate cancer two years postoperatively. In multivariate analysis, we found no significant difference in postoperative HRQOL and urinary continence recovery in patients with de-novo oligo-metastatic compared to patients with locally confined prostate cancer.

Abstract: (1) Background: local treatment of the primary tumor has become a valid therapeutic option in de-novo oligo-metastatic prostate cancer (PC). However, evidence regarding radical prostatectomy (RP) in this setting is still subpar, and the effect of cytoreductive RP on postoperative health-related quality of life (HRQOL) is still unclear. (2) Methods: for the current study, patients with de-novo oligo-metastatic PC (cM1-oligo), defined as $\leq 5$ bone lesions in the preoperative staging, were included, and matched cohorts using the variables age, body-mass index (BMI), and pT-stage were generated. Patient-reported outcome measures (PROMS) were assessed pre- and postoperatively using the validated EORTC-QLQ-C30, IIEF-5, and ICIQ-SF questionnaires. The primary endpoint for univariate and multivariable analysis was good general HRQOL defined by previously validated cut-off values. (3) Results: in total, 1268 patients ( $n=84$ (7\%) cM1-oligo) underwent RP between 2012 and 2020 at one tertiary care center. A matched cohort of 411 patients ( $n=79$ with oligo-metastatic bone disease (cM1-oligo) and $n=332$ patients without clinical indication of metastatic disease (cM0)) was created. The median follow-up was $25 \mathrm{mo}$. There was no significant difference in good general HRQOL rates between cM1-oligo-patients and cM0-patients before RP $(45.6 \%$ vs. 55.2\%, $p=$ $0.186)$, and at time of follow-up ( $44 \%$ vs. $56 \%, p=0.811$ ). Global health status (GHS) worsened significantly in cM0-patients compared to baseline $(-5, p=0.001)$, whereas GHS did not change significantly in cM1-oligo-patients $(+3.2, p=0.381)$. In multivariate analysis stratified for good erectile function (IIEF5 > 18; OR 5.722, 95\% CI 1.89-17.36, $p=0.002$ ) and continence recovery (OR 1.671, 95\% CI 1.03-2.70, $p=0.036)$, cM1-oligo was not an independent predictive feature for general HRQOL (OR $0.821,95 \%$ CI $0.44-1.53, p=0.536$ ). (4) Conclusions: in this large contemporary retrospective analysis, we observed no significant difference in HRQOL in patients with the oligometastatic bone disease after cytoreductive radical prostatectomy, when compared to patients with localized disease at time of surgery. 
Keywords: cytoreductive; HRQOL; metastatic prostate cancer

\section{Introduction}

In recent years, a plethora of systemic therapeutic options for patients with advanced prostate cancer have emerged [1-6]. Furthermore, large randomized trials showed a survival benefit of radiation therapy of the primary tumor in patients with low-volume metastatic disease [7-9]. These data led to the recommendation of current guidelines to offer androgen deprivation therapy (ADT) combined with radiotherapy of the prostate to patients presenting with low-volume metastatic prostate cancer as part of a multimodal therapy regime [10]. In contrast, evidence for surgical removal of the primary tumor (radical prostatectomy, RP) is sparse, and consequently, RP is not recommended by current guidelines outside of clinical trials. It has been described that RP significantly impacts postoperative health-related quality of life (HRQOL) in the respective patients. Hereby, studies assessing self-reported HRQOL showed that patients who underwent RP had a higher prevalence of urinary incontinence and erectile dysfunction compared to radiotherapy and observation, respectively [11]. In addition, it has been shown that the risk of subpar functional outcomes correlates with the local tumor stage [12]. To date, there is no analysis focused on HRQOL after cytoreductive RP. Considering the fact that many patients with de-novo metastatic bone-disease also present with locally advanced cancer but are otherwise asymptomatic, this raises the question of whether offering cytoreductive $\mathrm{RP}$ might cause unnecessary harm to the respective patients.

To address this paucity of data, we performed a comprehensive analysis of the effect of cytoreductive RP on the HRQOL in a contemporary and well-balanced cohort of patients with oligometastatic prostate cancer.

\section{Materials and Methods}

\subsection{Patient Population, Study Design, and Data Assessment}

Inclusion criteria for the current study encompassed: biopsy-proven prostate cancer, history of RP at one tertiary center and completed follow-up. Patients with preoperative ADT and pre-RP radiotherapy of the prostate were excluded from further analysis. Between July 2012 and September 2020, 1268 patients ( $n=800$ open retropubic, $n=468$ robot-assisted laparoscopic) fulfilled these criteria and were considered for analysis. Surgical standard procedures at our department have been described before [13]. A total of 84 of 1268 (7\%) patients had suspicion of oligometastatic bone disease (cM1-oligo) based on preoperative imaging at the time of RP. CM1-oligo was defined as $\leq 5$ bone lesions in the preoperative staging. Consequently, patients with nodal-only metastatic disease (cM1a), as well as visceral metastases (cM1c), did not undergo further analysis. All patients were informed about and consented in the experimental and individual multimodal therapeutic approach.

After approval by the local ethics committee (\#20-1022), PROMs were prospectively documented pre- and postoperatively. Hereby, questionnaires were sent by mail to eligible patients. Erectile function was assessed using the validated International Index of Erectile Function (IIEF-5), defining good erectile function with IIEF-5 score $\geq 18$ [14]. Urinary continence was assessed using the validated short form of the International Consultation on Incontinence Questionnaire (ICIQ-SF). With scores ranging from 0 to 21, higher scores indicate worsening of urinary continence [15]. Continence recovery was defined as the usage of up to one security pad per day. HRQOL was assessed using the validated European Organization for Research and Treatment of Cancer (EORTC) quality of life questionnaire (QLQ)-C30. According to EORTC guidelines, general HRQOL was assessed using the global health status (GHS) domain of the QLQ-C30 questionnaire. Good general HRQOL was defined as a GHS $\geq 70$ following previously published cut-off values [16]. For QLQ-C30 functioning scores, higher scores indicate better functioning. For QLQC30 symptom scores, higher scores indicate a greater impact on the respective symptom. 
Perioperative patient characteristics were assessed by analysis of the respective institutional medical records.

\subsection{Statistical Analysis}

Based on the clinical variables "age", "BMI," and "pT stage", 2 matched cohorts were generated. The 3 variables were chosen based on previously published studies indicating that these variables represent independent risk factors for postoperative impairment of HRQOL and functional outcome $[12,17,18]$. Due to the limited sample size of cM1-oligo patients, the matching process was limited to 3 variables and not expanded throughout all variables that showed significant differences in the unmatched cohort. Consequently, a matched cohort of 411 patients $(n=79$ with oligometastatic bone disease and $n=332$ without signs of metastatic disease) was created and considered for further analysis.

Testing of normal distribution was performed by the Shapiro-Wilk test. Statistical analysis was performed using SPSS V28 software (IBM, Armonk, NY, USA). Continuous variables were presented as means and standard deviation as well as median with the interquartile range as indicated. Categorical variables were presented as absolute numbers and percentages. Fisher's exact test and Chi-square test were used for univariate analysis of categorical data. The Mann-Whitney $\mathrm{U}$ test was used for univariate analysis of continuous data. The primary endpoint for univariate and multivariable analysis was good general HRQOL with a GHS $\geq 70$. For multivariable analysis, Cox regression models and binary logistic regression models were used. For univariate survival analyses, Kaplan-Meier curves were generated, and log-rank testing was performed. A $p$-value of $<0.05$ was considered statistically significant.

\section{Results}

\subsection{Patient Characteristics and Survival Outcomes}

Detailed patient characteristics of the unmatched and matched cohorts are displayed in Table 1.

Briefly, preoperative tumor burden was generally higher in the unmatched cM1-oligo cohort compared to unmatched cM0-patients with significantly higher median PSA levels, Gleason grade group $8-10$, and positive lymph node disease $(p<0.001$, respectively).

In the next step, a matched cohort was created as described above. The median follow-up for the entire cohort was 25 months.

Regarding the cM1-oligo cohort, 28 patients (35.4\%) were staged using PSMA-PET/Ct, whereas staging was based on conventional imaging in 51 patients $(64.6 \%)$. Regarding the cM0 cohort, 27 patients (9.7\%) were staged using PSMA-PET/Ct, whereas staging was based on conventional imaging in 192 patients $(68.8 \%)$. A total of 53 patients $(16 \%)$ of the cM0-cohort presented a low-risk profile and did not undergo preoperative staging.

Notably, the ratio of robot-assisted laparoscopic radical prostatectomies was significantly lower in cM1-oligo patients compared to cM0-patients $(12.7 \%$ vs. $34.9 \%, p<0.001)$. Furthermore, patients in the cM1-oligo subgroup showed significantly higher rates of history of TUR-P (12.7 vs. 3.0\%, $p=0.001)$ and a significantly higher rate of patients with an American Society of Anesthesiology (ASA) score of $\geq 3$ ( 60.9 vs. $44.6 \%, p=0.017$ ). There were no differences in rates of history of hernia surgery between both groups $(p=0.723)$ and the median number of lymph nodes removed during the surgical procedure $(p=0.124)$.

Regarding adjuvant and salvage therapies, radiotherapy rates $(22.6 \%$ vs. $24.1 \%$, $p=0.401$ ) after surgery did not vary significantly between cM1-oligo and cM0-patients. However, rates of perioperative androgen deprivation therapy were significantly higher for cM1-oligo patients ( 53.8 vs. $35.4 \%, p=0.016$ ), as well as rates novel anti-androgen therapies (10.1 vs. $2.7 \%, p=0.003)$, and taxane-based chemotherapy (6.3 vs. $1.0 \%, p<0.001)$. Survival analysis revealed a significantly shorter estimated 5-year cancer-specific survival rate $(61 \%$ vs. $81 \%, p=0.006)$ and a shorter estimated 5 -year overall-survival rate ( $38 \%$ vs. $57 \%$, $p=0.004$ ) for cM1-oligo patients compared to cM0-patients (Figure S1a,b). 
Table 1. Perioperative patient characteristics, Bold values indicate $p$-values $<0.05$ and were considered statistically significant. Continuous values are presented as median and inter-quartile-range (IQR); categorical values are given as number ( $n$; \%) BMI: Body-Mass-Index; PSA preop: preoperative Prostate-specific Antigen level; LN: Lymphnode.

\begin{tabular}{|c|c|c|c|c|c|c|}
\hline & \multicolumn{3}{|c|}{ Unmatched Cohort } & \multicolumn{3}{|c|}{ Matched Cohort } \\
\hline & cM1-Oligo & cM0 & $p$ & cM1-Oligo & cM0 & $p$ \\
\hline No. of patients & 84 & 1184 & & 79 & 332 & \\
\hline Age, years (median, IQR) $\#$ & $66(59,73)$ & $69(66,77)$ & 0.039 & $66(60,72)$ & $68(60,73)$ & 0.672 \\
\hline BMI kg $/ \mathrm{m}^{2}$ (median, IQR) $\#$ & $25.7(24.0,27.8)$ & $26.1(24.3,28.7)$ & 0.154 & $25.7(24.0,28)$ & $26.2(24.3,29.1)$ & 0.158 \\
\hline PSA preop. ng/mL (median, IQR) & $25.2(6.5,63.3)$ & $8.3(5.9,13.6)$ & $<0.001$ & $21.1(6.4,51.0)$ & $10.9(6.8,20.2)$ & 0.024 \\
\hline Prostate volume $\mathrm{mL}$ (median, IQR) & $47(40,58)$ & $52(41,66)$ & 0.007 & $47.5(39.8,58.0)$ & $53.5(43.0,67.0)$ & 0.002 \\
\hline \multicolumn{7}{|l|}{ Gleason score $(n(\%))$} \\
\hline 6 & $0(0.0)$ & $137(11.5)$ & \multirow{6}{*}{$<0.001$} & $0(11.1)$ & $15(4.5)$ & \multirow{6}{*}{$<0.001$} \\
\hline $7 a$ & $3(3.6)$ & $445(37.6)$ & & $3(3.8)$ & $63(19.0)$ & \\
\hline $7 \mathrm{~b}$ & $10(11.9)$ & $270(22.8)$ & & $9(11.4)$ & $82(24.7)$ & \\
\hline 8 & $18(21.4)$ & $139(11.8)$ & & $17(21.5)$ & $54(16.3)$ & \\
\hline 9 & $46(54.8)$ & $180(15.2)$ & & $42(53.2)$ & $105(31.6)$ & \\
\hline 10 & $7(8.3)$ & $13(1.1)$ & & $8(10.2)$ & $13(3.9)$ & \\
\hline \multicolumn{7}{|l|}{ pT stage $(n(\%))^{\#}$} \\
\hline pT2a & $0(0.0)$ & $62(5.3)$ & \multirow{6}{*}{0.001} & $0(0.0)$ & $0(0.0)$ & \multirow{6}{*}{0.221} \\
\hline $\mathrm{pT} 2 \mathrm{~b}$ & $0(0.0)$ & $24(2.0)$ & & $0(0.0)$ & $0(0.0)$ & \\
\hline pT2c & $10(11.9)$ & $616(52.1)$ & & $10(12.7)$ & $60(18.1)$ & \\
\hline pT3a & $15(17.9)$ & $265(22.4)$ & & $15(19.0)$ & $88(26.5)$ & \\
\hline pT3b & $54(64.3)$ & $211(17.8)$ & & $52(65.8)$ & $178(53.6)$ & \\
\hline pT4 & $5(6.0)$ & $5(0.4)$ & & $2(2.5)$ & $6(1.8)$ & \\
\hline Nerve sparing $(n(\%))$ & $14(16.7)$ & $859(72.6)$ & $<0.001$ & $13(16.5)$ & $183(55.1)$ & $<0.001$ \\
\hline Robot-assisted RP ( $n(\%))$ & $10(11.9)$ & $458(38.7)$ & $<0.001$ & $10(12.7)$ & $116(34.9)$ & $<0.001$ \\
\hline Positive surgical margin $(n(\%))$ & $64(76.2)$ & $322(27.2)$ & $<0.001$ & $59(74.7)$ & $165(49.7)$ & $<0.001$ \\
\hline Lymph node involvement $(n(\%))$ & $46(54.8)$ & $343(29.0)$ & $<0.001$ & $41(51.9)$ & $112(33.7)$ & 0.003 \\
\hline LN removed (median, IQR) & & & & $10(6,13)$ & $11(6,18)$ & 0.124 \\
\hline positive LN (median, IQR) & & & & $1(0,3)$ & $1(0,1)$ & 0.059 \\
\hline PSA postop. ng/mL (median, IQR) & & & & $9.5(2.0,70.3)$ & $0.0(0.0,0.0)$ & $<0.001$ \\
\hline
\end{tabular}

" matched variables.

\subsection{Functional Outcomes}

Pre- and postoperatively assessed functional outcomes are displayed in Table 2. In summary, we observed significantly lower total IIEF-5 scores in cM1-oligo patients compared to cM0-patients preoperatively as well as at the time of follow-up (8.5 (SD 10.2) vs. 11.3 (9.9), $p=0.022 ; 1.3$ (4.2) vs. 3.5 (6.2), $p<0.001)$. Regarding urinary continence, ICIQ-SF scores, amount of daily used pads, and rate of continence recovery did not vary significantly between cM1-oligo patients and cM0-patients. Preoperative ICIQ-SF score was 2.3 (SD 4.6) for cM1-oligo patients and 1.1 (2.6) for cM0-patients. Postoperatively, scores raised in both subgroups to 6.4 (5.7) for cM1-oligo patients and 6.4 (5.2) for cM0 patients. The median number of daily pad use at the time of follow-up was 1.6 (SD 2.5) for cM1-oligo patients and 1.2 (1.7) for cM0 patients. At the time of follow-up, 66\% of cM1oligo patients regained continence recovery compared to $72 \%$ of cM0 patients. Analysis of time to continence rehabilitation revealed no significant difference between cM1-oligo and cM0 patients $(p=0.773)$ (Figure 1$)$.

\subsection{Health-Related Quality of Life}

General HRQOL assessed by GHS, symptoms as well as functioning subdomains, and financial difficulty scales are summarized in Table 3.

Preoperatively, cM1-oligo patients expressed significantly more severe symptoms concerning pain and fatigue compared to cM0-patients (18.4 (SD 27) vs. 11.2 (20.7), $p=0.031$; $23.6(26.4)$ vs. 13.7 (16.7), $p=0.012$ ). In addition, cM1-oligo patients expressed higher financial difficulties scores compared to cM0-patients (11.1 (SD 23.8) vs. 2.7 (10.7), $p<0.001$ ). Regarding preoperative functioning subdomains, cM1-oligo patients showed significantly 
lower role functioning (83.6 (SD 26.4) vs. 91.9 (19.4), $p=0.004$ ), emotional (65.8 (SD 24.3) vs. 74.2 (20.9), $p=0.014$ ) and social functioning (77.2 (SD 25.1) vs. 85.5 (21.6), $p=0.007$ ) compared to cM0-patients. Regarding general HRQOL, cM1-oligo patients showed significantly lower preoperative GHS scores compared to cM0-patients (63.6 (SD 20.1) vs. 71.8 (S20.7), $p=0.004$ ). The rate of patients with good general HRQOL (GHS $\geq 70$ ) did not vary significantly between both groups.

Table 2. Pre- and postoperative functional outcomes after a median follow-up of 25 months. Bold values indicate $p$-values $<0.05$ and were considered statistically significant. Continuous values are presented as median and standard deviation (SD). IIEF-5: International Index of Erectile Function; ICIQ-SF: International Consultation on Incontinence Questionnaire -Short Form.

\begin{tabular}{ccccccc}
\hline & \multicolumn{3}{c}{ T0 } & \multicolumn{3}{c}{ Follow-Up } \\
\cline { 2 - 7 } & cM1-Oligo & cM0 & $p$ & cM1-Oligo & cM0 & $p$ \\
\hline Erectile function & & & & & & \\
IIEF-5 score [mean, SD] & $8.5(10.2)$ & $11.3(9.9)$ & 0.022 & $1.3(4.2)$ & $3.5(6.2)$ & $<0.001$ \\
IIEF-5 score 18 or more [\%] & 26.8 & 37.2 & 0.135 & 2.0 & 6.8 & 0.196 \\
Urinary continence & & & & & & \\
ICIQ-SF score [mean, SD] & $2.3(4.6)$ & $1.1(2.6)$ & 0.081 & $6.4(5.7)$ & $6.4(5.2)$ & 0.970 \\
Daily pad usage [mean, SD] & n.a. & n.a. & n.a. & $1.6(2.5)$ & $1.2(1.7)$ & 0.195 \\
Continence recovery [\%] & n.a. & n.a. & n.a. & 66.0 & 72.0 & 0.383 \\
\hline
\end{tabular}

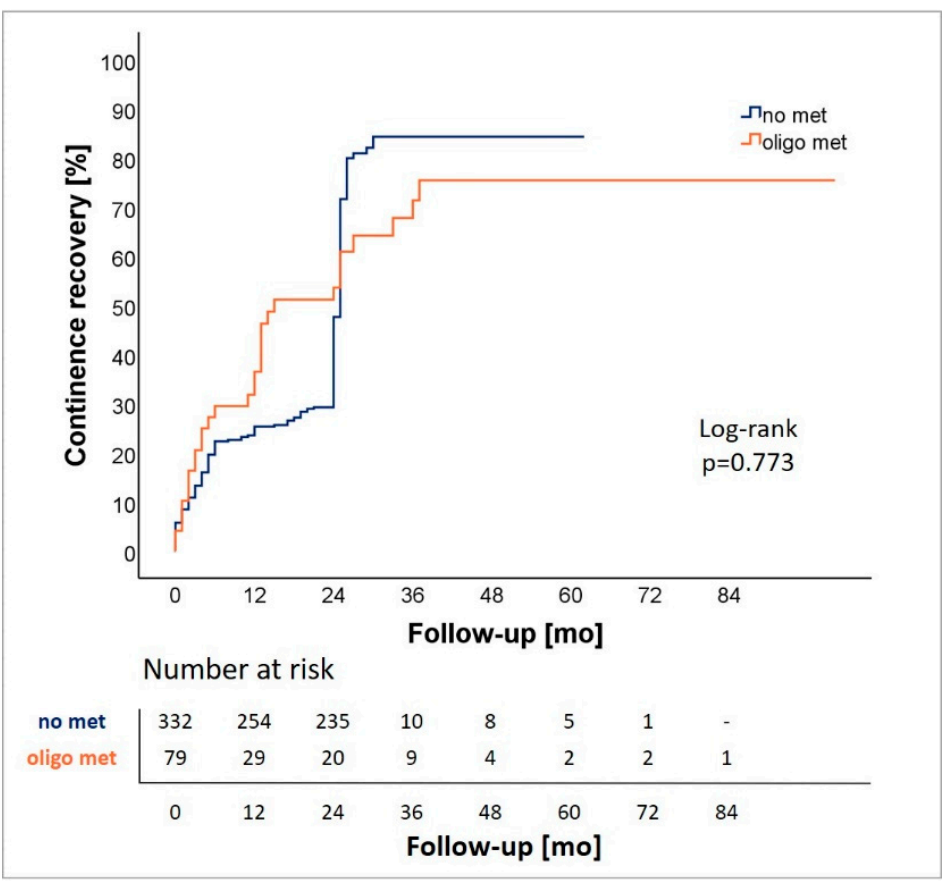

Figure 1. Analysis of continence recovery for patients with (oligo met) and without oligometastatic disease (no met).

At a median follow-up of 25 months, we observed significantly worse emotional functioning for cM1-oligo compared to cM0 patients (67.4 (SD 23.8) vs. 75 (22), $p=0.031$ ). In addition, cM1-oligo-patients reported to be significantly less sexually active compared cM0-patients) 22.8 (SD 24.9) vs. 37.5 (29.9), $p=0.001$ ). There were no significant differences regarding the remaining symptoms and functioning subscales as well as general HRQOL based on GHS.

Analysis of net changes compared to preoperative baseline is displayed in Figure 2 and Table S1. Briefly, we observed no significant change in GHS in cM1-oligo patients $(+3.2$, $p=0.381)$, but a significant decrease in cM0-patients $(-5, p=0.001)$. 
Table 3. Pre- and postoperative patient-reported HQOL, assessed by the validated EORTC QLQ-C30 questionnaire. Bold values indicate $p$-values $<0.05$ and were considered statistically significant. Continuous values are presented as median and standard deviation (SD). HRQOL: health-related quality of life; EORTC-QLQ-C30: European Organization for Research and Treatment of Cancer quality of life questionnaire-Cancer 30; QLQ-PR25: European Organization for Research and Treatment of Cancer quality of life questionnaire-Prostate 25.

\begin{tabular}{|c|c|c|c|c|c|c|c|}
\hline & & \multicolumn{6}{|c|}{ Mean (SD) EORTC QLQ C30 Score } \\
\hline & & \multicolumn{3}{|c|}{ T0 } & \multicolumn{3}{|c|}{ Follow-Up } \\
\hline \multirow{18}{*}{ QLQ-C 30} & & cM1-Oligo & cM0 & $p$ & cM1-Oligo & cM0 & $p$ \\
\hline & Symptom scale & & & & & & \\
\hline & Dyspnoea & $7.1(16.3)$ & $7.7(18.7)$ & 0.959 & $12.2(21.2)$ & $20.1(26.6)$ & 0.047 \\
\hline & Pain & $18.4(27.0)$ & $11.2(20.7)$ & 0.031 & $16.3(24.9)$ & $15.3(24.6)$ & 0.503 \\
\hline & Fatigue & $23.6(26.4)$ & $13.7(16.7)$ & 0.012 & $31.9(25.1)$ & $25.5(25.3)$ & 0.052 \\
\hline & Insomnia & $23.0(33.9)$ & $18.7(26.1)$ & 0.706 & $28.6(37.3)$ & $27.8(30.4)$ & 0.590 \\
\hline & $\begin{array}{l}\text { Appetite } \\
\text { loss }\end{array}$ & $4.8(13.4)$ & $3.3(12.7)$ & 0.250 & $7.5(18.3)$ & $4.4(13.3)$ & 0.272 \\
\hline & Nausea/vomiting & $0.1(2.2)$ & $1.0(4.2)$ & 0.069 & $1.4(5.7)$ & $3.5(10.3)$ & 0.154 \\
\hline & Constipation & $7.9(23.1)$ & $5.7(16.4)$ & 0.949 & $15.6(27.3)$ & $11.2(22.8)$ & 0.259 \\
\hline & Diarrhoea & $7.6(18.9)$ & $6.9(15.7)$ & 0.752 & $12.9(25.3)$ & $12.0(21.4)$ & 0.879 \\
\hline & $\begin{array}{l}\text { Financial difficulty scale } \\
\text { Functioning scale }\end{array}$ & $11.1(23.8)$ & $2.7(10.7)$ & $<0.001$ & $13.2(26.4)$ & $8.0(18.8)$ & 0.207 \\
\hline & Physical & $90.4(15.6)$ & $93.7(12.7)$ & 0.130 & $79.4(23.3)$ & $86.1(16.8)$ & 0.177 \\
\hline & Role & $83.6(26.4)$ & $91.9(19.4)$ & 0.004 & $75.5(29.9)$ & $77.0(26.4)$. & 0.984 \\
\hline & Cognitive & $86.8(18.3)$ & $89.6(16.6)$ & 0.272 & $80.9(25.3)$ & $85.3(19.4)$ & 0.398 \\
\hline & Emotional & $65.8(24.3)$ & $74.2(20.9)$ & 0.014 & $67.4(23.8)$ & $75.0(22.0)$ & 0.031 \\
\hline & Social & $77.2(25.1)$ & 85.5 (21.6) & 0.007 & $71.7(29.1)$ & $73.2(28.6)$ & 0.702 \\
\hline & Global health status & $63.6(20.1)$ & $71.8(20.7)$ & 0.004 & $66.8(20.8)$ & $66.8(21.5)$ & 0.959 \\
\hline & $\begin{array}{l}\text { Global health status } \geq \\
70[\%]\end{array}$ & 45.6 & 55.2 & 0.186 & 44.0 & 56.0 & 0.811 \\
\hline \multirow{6}{*}{ QLQ-PR25 } & Urinary symptoms & & & & $27.4(17.5)$ & $29.2(18.9)$ & 0.707 \\
\hline & $\begin{array}{l}\text { Incontinence } \\
\text { aid }\end{array}$ & & & & $29.5(28.8)$ & $39.5(34.1)$ & 0.185 \\
\hline & $\begin{array}{l}\text { Bowel } \\
\text { symptoms }\end{array}$ & & & & $7.6(12.9)$ & $8.9(13.4)$ & 0.522 \\
\hline & Treatment symptoms & & & & $24.7(15.9$ & $20.7(16.9)$ & 0.058 \\
\hline & Sexually active & & & & $22.8(24.9)$ & $37.5(29.9)$ & 0.001 \\
\hline & $\begin{array}{l}\text { Sexual } \\
\text { functioning }\end{array}$ & & & & $55.4(23.0)$ & $50.8(17.6)$ & 0.743 \\
\hline
\end{tabular}

\section{Net Baseline Changes QLQ Subscales}

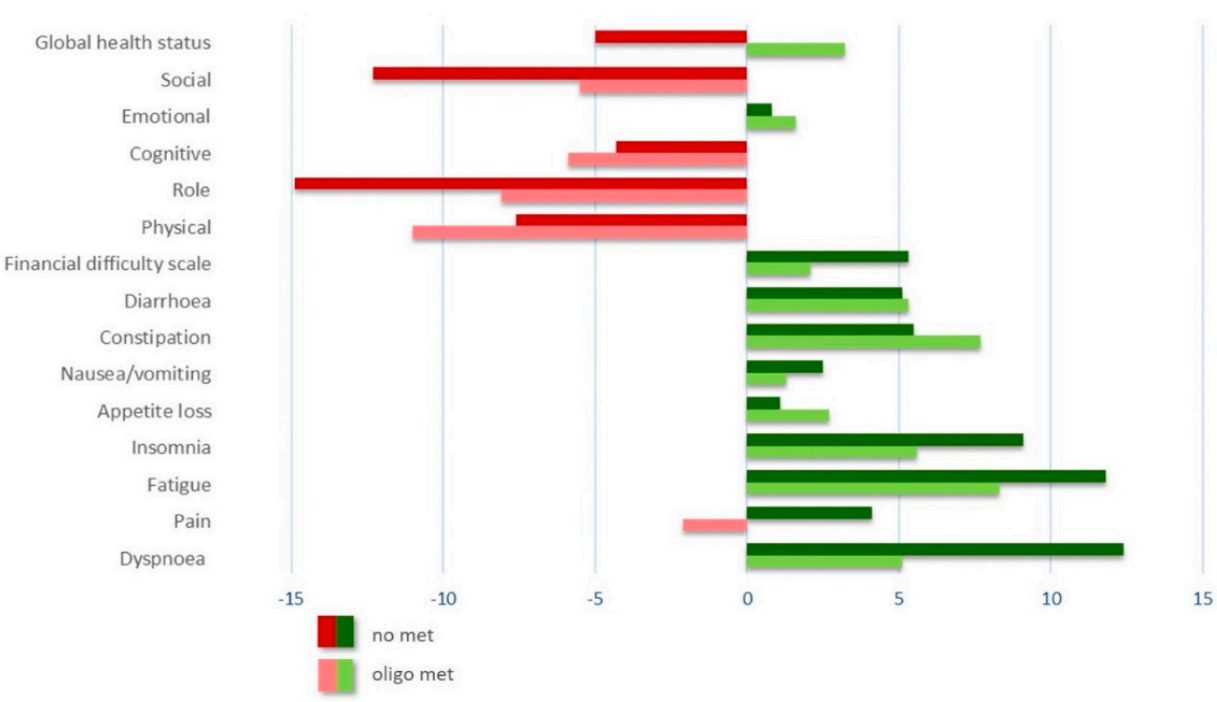

Figure 2. Trend illustration of net baseline changes between pre- and postoperative HRQOL. 
In the next step, we assessed the longitudinal time course of postoperative HRQOL outcomes in our matched patient cohorts. Detailed results of the respective QLQ-C30 and PR-25 subdomains at follow-up time points of 3, 12, 24, and 36 months postoperatively are summarized in Table S2. Briefly, we found comparable HRQOL functioning subdomains courses throughout a follow-up period of 36 months. Addressing general HRQOL based on the QLQ-C30 GHS, we found increased mean GHS scores for cM0 patients 12 months postoperatively (71.5 vs. $64.2, p=0.027)$, without any statistically significant differences at the remaining time points (Figure 3). Regarding the PR25 add-on, we found significantly increased sexually active scores for cM0 patients 24 months postoperatively, without any statistically significant differences during the remaining postoperative time course (Table S2).

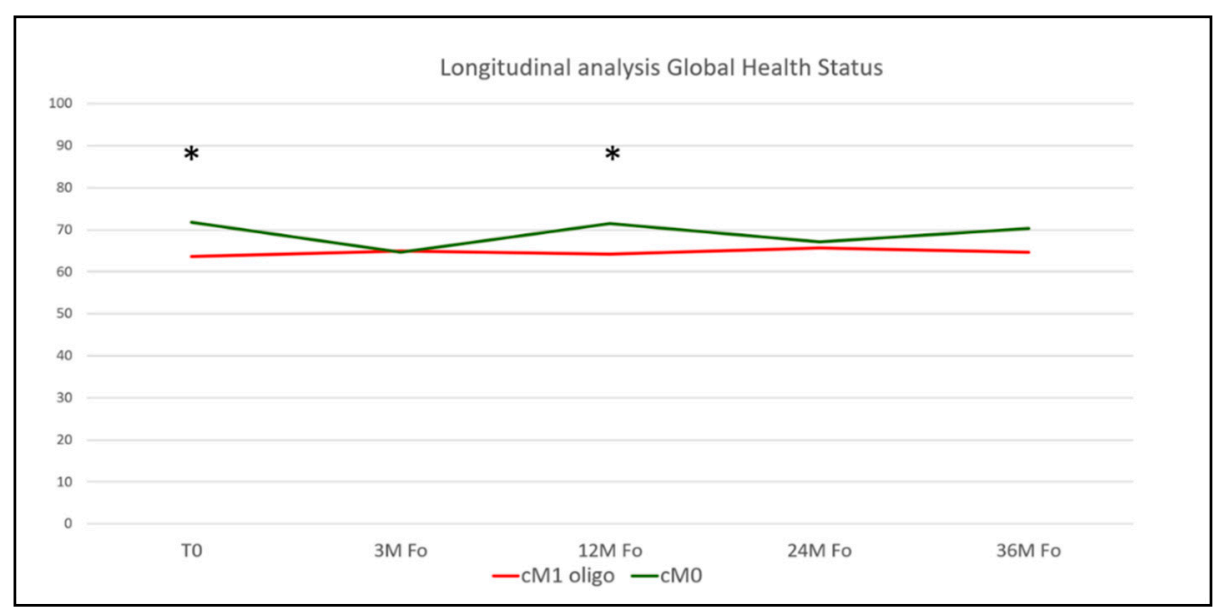

Figure 3. Longitudinal analysis of postoperative patient-reported Global Health Status, assessed by the validated EORTC QLQ-C30 questionnaire. Asterisks indicate $p$-values $<0.05$ and were considered statistically significant. EORTC-QLQ-C30: European Organization for Research and Treatment of Cancer quality of life questionnaire-Cancer 30.

In multivariate analysis for the primary endpoint good general HRQOL (defined as a GHS $\geq 70$ following previously published cut-off values) stratified for good erectile function (IIEF5 > 18; OR 5.722, 95\% CI 1.89-17.36, $p=0.002$ ) and continence recovery (OR 1.671, 95\% CI 1.03-2.70, $p=0.036$ ), presence of oligo-metastatic bone disease did not represent a significant predictive feature for good general HRQOL at time of follow-up (OR $0.821,95 \%$ CI $0.44-1.53, p=0.536$ ) (Table 4 ).

Table 4. Multivariable analysis assessing the primary endpoint good general HRQOL (defined as GHS of $\geq 70$ ). Bold values indicate $p$-values $<0.05$ and were considered statistically significant. Net change to preoperative status is presented, HRQOL: health-related quality of life; IIEF-5: International Index of Erectile Function; RP: Radical prostatectomy GHS: Global-Health status according to the European Organization for Research and Treatment of Cancer quality of life questionnaire-Cancer 30.

\begin{tabular}{cccccc}
\hline \multicolumn{7}{c}{ Multivariate Logistic Regression for Good HRQOL } \\
\hline $\begin{array}{c}\text { Predictive Feature for Good } \\
\text { HRQOL }\end{array}$ & $\begin{array}{l}\text { Regression } \\
\text { Coefficient }\end{array}$ & $\begin{array}{l}\text { Odds } \\
\text { Ratio }\end{array}$ & 95\% CI & $p$ Value \\
\hline & & & Lower & Upper \\
\hline cM1-oligo (yes vs. no) & -0.197 & 0.821 & 0.44 & 1.53 & 0.536 \\
IIEF-5 18 or more (yes vs. no) & 1.744 & 5.722 & 1.89 & 17.36 & 0.002 \\
Continence recovery (yes vs. no) & 0.514 & 1.671 & 1.03 & 2.70 & 0.036 \\
Nerve-sparing (yes vs. no) & 0.384 & 1.468 & 0.93 & 2.32 & 0.101 \\
Robot-assisted RP (yes vs. no) & -0.448 & 0.639 & 0.40 & 1.03 & 0.067 \\
\hline
\end{tabular}




\section{Discussion}

"First, do not harm" has been a credo for every physician's action for many centuries now. This is crucial when it comes to experimental therapeutic strategies such as cytoreductive RP for oligo-metastatic prostate cancer. Regarding the paucity of data on postoperative HRQOL following cytoreductive RP, we aimed to address this hypothesis in a contemporary well-balanced and adequately large patient cohort comparing patients undergoing cytoreductive radical prostatectomy to a control group of patients with localized disease.

Multiple studies showed a significant impact on postoperative HRQOL following $\mathrm{RP}$ for localized prostate cancer. The PROTECT study randomly assigned 1643 patients with localized prostate cancer to either monitoring, RP, or radiotherapy [19]. Donovan et al. evaluated HRQOL and functional outcomes with EORTC-QLQ-C30 and ICIQ questionnaires among others. The authors showed that RP had the greatest negative effect on urinary continence compared to monitoring and radiotherapy after a median followup of 24 months [20]. The higher mean ICIQ scores in the current study might derive from a significantly lower tumor burden in the PROTECT study group (median PSA $4.7-4.9 \mathrm{ng} / \mathrm{mL}$, Gleason 6 in 76-78\% throughout the treatment arms) and indicate a more pronounced impact on functional outcomes by higher tumor burden. In the PROTECT study, GHS after 5-years of follow-up did not vary significantly between the three treatment arms. GHS scores in the current study are lower after 25 months of follow-up compared to PROTECT, possibly deriving from shorter follow-up and higher local tumor burden. Further studies did not observe a significant impact of the surgical technique (open retropubic vs. laparoscopic robot-assisted) on long-term HRQOL following RP [13].

With regards to more advanced disease stages, patients with newly diagnosed oligometastatic prostate cancer can be offered multiple treatment options besides conventional $\mathrm{ADT}$ and currently approved substances enzalutamide and apalutamide showed significantly prolonged overall survival compared to baseline ADT as standard of care in this setting [1,4-6].

In addition, analyses from the multi-arm STAMPEDE trial showed improved overall survival in patients with low metastatic burden treated with additional radiotherapy of the primary tumor [7]. In contrast, cytoreductive RP is rarely performed today, and evidence is still subpar and is based on retrospective analyses of large registry databases that showed improved overall survival in selected patients who underwent cytoreductive RP compared to conservative therapy regimes [21-24]. Another rationale for cytoreductive RP might be local symptom control. Up to $78 \%$ of patients with metastatic prostate cancer will suffer from local complications, such as bladder outlet obstruction, ureteric obstruction, and gross hematuria at some stage [25], and it has been shown that local treatment resulted in a reduced complication rate compared to best supportive care [24].

Furthermore, data on the impact of cytoreductive RP on HRQOL is sparse. Reichard et al. retrospectively analyzed a selected group of 14 patients and reported on pre- and postoperative functional outcomes by the urinary domain of the Expanded Prostate Cancer Index Composite (EPIC) and observed a decline after a maximum follow-up of three months [26].

In the current study, we provide a matched analysis of the impact of cytoreductive RP on HRQOL in a large contemporary cohort. To our knowledge, this resembles the largest evaluation of HRQOL after cytoreductive RP to date. Hereby, we did not find significant differences of postoperative HRQOL in patients with oligometastatic disease compared to a matched control cohort with localized disease.

In the (oligo)metastatic setting, Agarwal et al. assessed the impact of the treatment with apalutamide and ADT compared to ADT and placebo on HRQOL using the FACT-P questionnaire. The study cohort mainly comprised high-volume patients (up to 66\%) and only $18 \%$ of patients in the apalutamide arm had metachronous metastatic disease with prior local therapy. The authors showed that HRQOL did not vary between both groups regarding time-to-deterioration or net baseline changes [27]. In the ARCHES study, Stenzl et al. evaluated the effect of enzalutamide combination therapy on HRQOL in 
metastatic hormone-sensitive prostate cancer patients [28]. The treatment arm contained $38.3 \%(220 / 574)$ patients classified as low-volume disease, and $25.3 \%$ had previous local therapy. HRQOL was assessed using the EORTC QLQ-PR25 and FACT-P questionnaire. Interestingly, the authors observed increased FACT-P physical well-being scores for the control arm in a subgroup of patients with low-volume disease. However, according to predefined threshold measures, this was not considered clinically meaningful. Overall, the authors found no significant differences in time-to-deterioration of HRQOL or pain between both arms as assessed by EORTC QLQ-PR25 and FACT-P scores. Although the comparability of this conservative treatment approach and our study is limited, these data are consistent with our findings. Regarding net changes from baseline, both arms of the ARCHES study showed a decline in general HRQOL over the course of therapy. The ratio of patients with low-volume disease with worsening lack of energy from baseline to end of follow-up was $48.1 \%$ for the treatment arm and $41.3 \%$ for the placebo arm. Conversely, we observed no significant change in GHS in cM1-oligo patients $(+3.2, p=0.381)$, but a significant decrease in cM0-patients $(-5, p=0.001)$. Regarding functional outcomes, Stenzl et al. found a longer time-to-deterioration in sexual activity in the placebo arm compared to the treatment arm. Likewise, we observed significantly lower total IIEF-5 scores in cM1-oligo patients compared to cM0-patients preoperatively as well as at the time of follow-up. Furthermore, cM1-oligo patients in the current study showed significantly lower postoperative QLQ-PR25 sexual activity scores compared to cM0-patient.

The current study has several limitations. Due to the retrospective design, confound patient characteristics such as specific co-morbidities were not available in detail. In addition, different staging modalities, including bone scans as well as PSMA-PET/CT was used, which might lead to a potential bias when defining oligometastatic disease stages. Furthermore, distinct information on mpMRI findings as well as molecular biomarker test results has not been assessed in the current population even though it has been shown that both modalities can work hand in hand in diagnosis in advanced prostate cancer [29]. Apart from the retrospective design, we used the non-prostate-specific EORTC-QLQ-C30 questionnaire to address HRQOL of prostate cancer patients. However, the EORTC-QLQ-C30 questionnaire is frequently used among other tumor entities and surgeries and, therefore, provides robust and comparable data. To address the specific domains of interest, sexual function and urinary continence, we complemented our evaluation by the validated ICIQSF and IIEF-5 questionnaires as well as the prostate-specific PR25 add-on to the QLQ-C30 questionnaire. Another potential limitation of the current study is the relatively short median follow-up of 25 months, as other studies showed significant changes in terms of sexual function and urinary continence until several years after surgery [20]. Even though this study represents the largest evaluation of HRQOL after cytoreductive RP to our knowledge, the sub-cohort of cM1-oligo patients is still relatively small compared to cM0 patients, leading to a possible risk of underpowering. Due to limitations in sample size, not all tumor burden indicators such as Gleason grade and preoperative PSA value could be included in the matching process. However, these factors did not show to be a significant predictor of good general HRQOL after RP in univariate and multivariable analysis. Finally, it has to be addressed that not all patients underwent initial PSMA-PET/CT scans prior to RP, leading to potential understaging. However, it has to be emphasized that this limitation is also inherent to currently available data from phase 3 trials in this setting. For instance, the SWOG 1802 trial (NCT 03678025) is currently recruiting [30]. Hereby, patients with metastatic prostate cancer based on conventional or PSMA-PET/CT based imaging are randomized into local therapy (RP or radiotherapy of the prostate) arm or a control arm while both arms undergo standard of care systemic androgen deprivation therapies. However, the first results are expected in 2028, and evidence from real-life data analyses will be relevant until mature data from randomized trials is publicly available.

\section{Conclusions}

In the current study, we assess HRQOL outcomes after cytoreductive RP from a comprehensive and contemporary matched patient cohort during a follow-up period of up 
to 36 months between patients with or without oligometastatic prostate cancer. Hereby, we found no significant differences in general HRQOL and functional outcomes between both subgroups in univariate as well as multivariable analysis indicating that cytoreductive $\mathrm{RP}$ can be offered safely to patients without subpar HRQOL outcomes compared to patients undergoing RP for localized disease.

Supplementary Materials: The following are available online at https:/ /www.mdpi.com/article/10 .3390/cancers13225636/s1, Figure S1: a. Estimated 5-year cancer-specific survival (CSS), b. Estimated 5-year overall survival (OS). Table S1: Numerical analysis of net baseline changes of HRQOL preand postoperatively. Bold values indicate $p$ values $<0.05$ and were considered statistically significant. Net change to preoperative status is presented HRQOL: Health-related quality of life; QLQ-C30: European Organization for Research and Treatment of Cancer quality of life questionnaire-Cancer 30., Table S2: Longitudinal analysis of postoperative patient-reported HRQOL, assessed by the validated EORTC QLQ-C30 questionnaire. Bold values indicate $p$ values $<0.05$ and were considered statistically significant. Continuous values are presented as median and standard-deviation (SD). HRQOL: Health-related quality of life; EORTC-QLQ-C30: European Organization for Research and Treatment of Cancer quality of life questionnaire-Cancer 30; QLQ-PR25: European Organization for Research and Treatment of Cancer quality of life questionnaire-Prostate 25.

Author Contributions: Conceptualization, M.C., A.K. and C.G.S.; methodology, M.C., L.S., T.W. and A.K.; software, T.W. and A.B.; validation, M.C., A.K. and C.G.S.; formal analysis, M.C., L.S., T.W. and A.K.; investigation, M.C., L.S., M.A., A.B., V.W., C.G.S., T.W., A.K.; resources, M.C., L.S. and A.K.; data curation, M.C., L.S. and A.K.; writing-original draft preparation, M.C., T.W. and A.K.; writing-review and editing, M.C., L.S., M.A., A.B., V.W., C.G.S., T.W., A.K.; visualization, M.C., T.W. and A.K.; supervision, C.G.S.; project administration, M.C., T.W. and A.K.; funding acquisition, C.G.S. All authors have read and agreed to the published version of the manuscript.

Funding: This research received no external funding.

Institutional Review Board Statement: The study was conducted according to the guidelines of the Declaration of Helsinki and approved by the Ethics Committee of Ludwig-Maximilians University Munich (protocol code \#20-0769; date of approval: 10/15/2020).

Informed Consent Statement: Informed consent was obtained from all subjects involved in the study.

Conflicts of Interest: The authors declare no conflict of interest.

\section{References}

1. Fizazi, K.; Tran, N.; Fein, L.; Matsubara, N.; Rodriguez-Antolin, A.; Alekseev, B.Y.; Özgüroglu, M.; Ye, D.; Feyerabend, S.; Protheroe, A.; et al. Abiraterone plus Prednisone in Metastatic, Castration-Sensitive Prostate Cancer. N. Engl. J. Med. 2017, 377, 352-360. [CrossRef] [PubMed]

2. James, N.D.; Spears, M.R.; Sydes, M.R. Abiraterone in metastatic prostate cancer. N. Engl. J. Med. 2017, 377, 1696-1697. [PubMed]

3. Armstrong, A.J.; Szmulewitz, R.Z.; Petrylak, D.P.; Holzbeierlein, J.; Villers, A.; Azad, A.; Alcaraz, A.; Alekseev, B.; Iguchi, T.; Shore, N.D.; et al. ARCHES: A Randomized, Phase III Study of Androgen Deprivation Therapy With Enzalutamide or Placebo in Men With Metastatic Hormone-Sensitive Prostate Cancer. J. Clin. Oncol. 2019, 37, 2974-2986. [CrossRef] [PubMed]

4. Chi, K.N.; Agarwal, N.; Bjartell, A.; Chung, B.H.; Gomes, A.J.P.D.S.; Given, R.; Soto, A.J;; Merseburger, A.S.; Özgüroglu, M.; Uemura, H.; et al. Apalutamide for Metastatic, Castration-Sensitive Prostate Cancer. N. Engl. J. Med. 2019, 381, 13-24. [CrossRef] [PubMed]

5. Davis, I.D.; Martin, A.J.; Stockler, M.R.; Begbie, S.; Chi, K.N.; Chowdhury, S.; Coskinas, X.; Frydenberg, M.; Hague, W.E.; Horvath, L.G.; et al. Enzalutamide with standard first-line therapy in metastatic prostate cancer. N. Engl. J. Med. 2019, 381, 121-131. [CrossRef]

6. Sweeney, C.J.; Chen, Y.-H.; Carducci, M.; Liu, G.; Jarrard, D.F.; Eisenberger, M.; Wong, Y.-N.; Hahn, N.; Kohli, M.; Cooney, M.M.; et al. Chemohormonal Therapy in Metastatic Hormone-Sensitive Prostate Cancer. N. Engl. J. Med. 2015, 373, 737-746. [CrossRef]

7. Parker, C.C.; James, N.D.; Brawley, C.D.; Clarke, N.W.; Hoyle, A.P.; Ali, A.; Ritchie, A.W.S.; Attard, G.; Chowdhury, S.; Cross, W.; et al. Radiotherapy to the primary tumour for newly diagnosed, metastatic prostate cancer (STAMPEDE): A randomised controlled phase 3 trial. Lancet 2018, 392, 2353-2366. [CrossRef]

8. Burdett, S.; Boevé, L.M.; Ingleby, F.C.; Fisher, D.; Rydzewska, L.H.; Vale, C.; van Andel, G.; Clarke, N.W.; Hulshof, M.C.; James, N.D.; et al. Prostate Radiotherapy for Metastatic Hormone-sensitive Prostate Cancer: A STOPCAP Systematic Review and Meta-analysis. Eur. Urol. 2019, 76, 115-124. [CrossRef] [PubMed] 
9. Boevé, L.M.; Hulshof, M.C.; Vis, A.N.; Zwinderman, A.H.; Twisk, J.W.; Witjes, W.P.; Delaere, K.P.; Van Moorselaar, R.J.A.; Verhagen, P.C.; Van Andel, G. Effect on Survival of Androgen Deprivation Therapy Alone Compared to Androgen Deprivation Therapy Combined with Concurrent Radiation Therapy to the Prostate in Patients with Primary Bone Metastatic Prostate Cancer in a Prospective Randomised Clinical Trial: Data from the HORRAD Trial. Eur. Urol. 2019, 75, 410-418.

10. Mottet, N.; Bellmunt, J.; Briers, E.; Bolla, M.; Bourke, L.; Cornford, P.; De Santis, M.; Henry, A.; Joniau, S.; Lam, T.; et al. EauEstro-Esur-Siog guidelines on prostate cancer. Edn. Presented at the Eau Annual Congress, Amsterdam, The Netherlands, 17-21 July 2020; EAU Guidelines Office: Arnhem, the Netherlands, 2020.

11. Lardas, M.; Liew, M.; Bergh, R.C.V.D.; De Santis, M.; Bellmunt, J.; Broeck, T.V.D.; Cornford, P.; Cumberbatch, M.G.; Fossati, N.; Gross, T.; et al. Quality of Life Outcomes after Primary Treatment for Clinically Localised Prostate Cancer: A Systematic Review. Eur. Urol. 2017, 72, 869-885. [CrossRef]

12. Heesakkers, J.; Farag, F.; Bauer, R.M.; Sandhu, J.; De Ridder, D.; Stenzl, A. Pathophysiology and Contributing Factors in Postprostatectomy Incontinence: A Review. Eur. Urol. 2017, 71, 936-944. [CrossRef] [PubMed]

13. Kretschmer, A.; Bischoff, R.; Chaloupka, M.; Jokisch, F.; Westhofen, T.; Weinhold, P.; Strittmatter, F.; Becker, A.; Buchner, A.; Stief, C.G. Health-related quality of life after open and robot-assisted radical prostatectomy in low- and intermediate-risk prostate cancer patients: A propensity score-matched analysis. World J. Urol. 2020, 38, 3075-3083. [CrossRef] [PubMed]

14. Mandel, P.; Kretschmer, A.; Chandrasekar, T.; Nguyen, H.; Buchner, A.; Stief, C.G.; Tilki, D. The effect of BMI on clinicopathologic and functional outcomes after open radical prostatectomy. Urol. Oncol. Semin. Orig. Investig. 2014, 32, 297-302. [CrossRef] [PubMed]

15. Avery, K.; Donovan, J.; Peters, T.J.; Shaw, C.; Gotoh, M.; Abrams, P. ICIQ: A brief and robust measure for evaluating the symptoms and impact of urinary incontinence. Neurourol. Urodyn. 2004, 23, 322-330. [CrossRef] [PubMed]

16. Snyder, C.F.; Blackford, A.L.; Okuyama, T.; Akechi, T.; Yamashita, H.; Toyama, T.; Carducci, M.A.; Wu, A.W. Using the EORTCQLQ-C30 in clinical practice for patient management: Identifying scores requiring a clinician's attention. Qual. Life Res. 2013, 22, 2685-2691. [CrossRef] [PubMed]

17. Anast, J.W.; Sadetsky, N.; Pasta, D.; Bassett, W.W.; Latini, D.; DuChane, J.; Chan, J.M.; Cooperberg, M.; Carroll, P.R.; Kane, C.J. The impact of obesity on health related quality of life before and after radical prostatectomy (data from Capsure). J. Urol. 2005, 173, 1132-1138. [CrossRef]

18. Brajtbord, J.S.; Punnen, S.; Cowan, J.E.; Welty, C.J.; Carroll, P.R. Age and Baseline Quality of Life at Radical Prostatectomy-Who Has the Most to Lose? J. Urol. 2014, 192, 396-401. [CrossRef]

19. Hamdy, F.C.; Donovan, J.L.; Lane, J.A.; Mason, M.; Metcalfe, C.; Holding, P.; Davis, M.; Peters, T.; Turner, E.; Martin, R.; et al. 10-Year Outcomes after Monitoring, Surgery, or Radiotherapy for Localized Prostate Cancer. N. Engl. J. Med. 2016, 375, 1415-1424. [CrossRef]

20. Donovan, J.L.; Hamdy, F.C.; Lane, J.A.; Mason, M.; Metcalfe, C.; Walsh, E.; Blazeby, J.; Peters, T.; Holding, P.; Bonnington, S.; et al. Patient-Reported Outcomes after Monitoring, Surgery, or Radiotherapy for Prostate Cancer. N. Engl. J. Med. 2016, 375, 1425-1437. [CrossRef]

21. Gratzke, C.; Engel, J.; Stief, C.G. Role of Radical Prostatectomy in Metastatic Prostate Cancer: Data from the Munich Cancer Registry. Eur. Urol. 2014, 66, 602-603. [CrossRef]

22. Culp, S.H.; Schellhammer, P.F.; Williams, M.B. Might Men Diagnosed with Metastatic Prostate Cancer Benefit from Definitive Treatment of the Primary Tumor? A SEER-Based Study. Eur. Urol. 2014, 65, 1058-1066. [CrossRef] [PubMed]

23. Sooriakumaran, P.; Nyberg, T.; Akre, O.; Widmark, A.; Hamdy, F.; Graefen, M.; Carlsson, S.; Steineck, G.; Wiklund, N.P. Survival Among Men at High Risk of Disseminated Prostate Cancer Receiving Initial Locally Directed Radical Treatment or Initial Androgen Deprivation Therapy. Eur. Urol. 2017, 72, 345-351. [CrossRef] [PubMed]

24. Tilki, D.; Pompe, R.S.; Bandini, M.; Marchioni, M.; Kretschmer, A.; Tian, Z.; Karakiewicz, I.P.; Evans, C.P. Local treatment for metastatic prostate cancer: A systematic review. Int. J. Urol. 2018, 25, 390-403. [CrossRef] [PubMed]

25. Patrikidou, A.; Brureau, L.; Casenave, J.; Albiges, L.; Di Palma, M.; Patard, J.-J.; Baumert, H.; Blanchard, P.; Bossi, A.; Kitikidou, K.; et al. Locoregional symptoms in patients with de novo metastatic prostate cancer: Morbidity, management, and disease outcome. Urol. Oncol. Semin. Orig. Investig. 2015, 33, 202.e9-202.e17. [CrossRef] [PubMed]

26. Reichard, C.A.; Gregg, J.R.; Achim, M.F.; Aparicio, A.M.; Pettaway, C.A.; Pisters, L.L.; Ward, J.; Davis, J.W.; Chapin, B.F. Radical Prostatectomy in Metastatic Castration-resistant Prostate Cancer: Feasibility, Safety, and Quality of Life Outcomes. Eur. Urol. 2018, 74, 140-143. [CrossRef]

27. Agarwal, N.; McQuarrie, K.; Bjartell, A.; Chowdhury, S.; Gomes, A.J.P.D.S.; Chung, B.H.; Özgüroglu, M.; Soto Álvaro, J.; Merseburger, A.S.; Uemura, H.; et al. Health-related quality of life after apalutamide treatment in patients with metastatic castration-sensitive prostate cancer (TITAN): A randomised, placebo-controlled, phase 3 study. Lancet Oncol. 2019, 20, 1518-1530. [CrossRef]

28. Stenzl, A.; Dunshee, C.; De Giorgi, U.; Alekseev, B.; Iguchi, T.; Szmulewitz, R.Z.; Flaig, T.W.; Tombal, B.; Morlock, R.; Ivanescu, C.; et al. Effect of Enzalutamide plus Androgen Deprivation Therapy on Health-related Quality of Life in Patients with Metastatic Hormone-sensitive Prostate Cancer: An Analysis of the ARCHES Randomised, Placebo-controlled, Phase 3 Study. Eur. Urol. 2020, 78, 603-614. [CrossRef] 
29. Ferro, M.; Crocetto, F.; Bruzzese, D.; Imbriaco, M.; Fusco, F.; Longo, N.; Napolitano, L.; La Civita, E.; Cennamo, M.; Liotti, A.; et al. Prostate Health Index and Multiparametric MRI: Partners in Crime Fighting Overdiagnosis and Overtreatment in Prostate Cancer. Cancers 2021, 13, 4723. [CrossRef] [PubMed]

30. ClinicalTrials.gov. Identifier: Nct03678025, Standard Systemic Therapy with or without Definitive Treatment in Treating Participants with Metastatic Prostate Cancer; National Library of Medicine (US): Bethesda, MD, USA, 2018. 